René Unda Lara: «El movimiento que lideró Rafael Correa

fue de una participación social inédita" [entrevista]

Sofia Bernat, Sol Logroño, Emiliano Sánchez Narvarte

Tram[p]as de la comunicación y la cultura (N. ${ }^{\circ} 83$ ), e034, abril-septiembre 2018

ISSN 2314-274X | https://doi.org/10.24215/2314274Xe034

http://perio.unlp.edu.ar/ojs/index.php/trampas

FPyCS | Universidad Nacional de La Plata

La Plata | Buenos Aires | Argentina

\title{
RENÉ UNDA LARA: \\ «EL MOVIMIENTO QUE LIDERÓ RAFAEL CORREA \\ FUE DE UNA PARTICIPACIÓN SOCIAL INÉDITA»
}

RENÉ UNDA LARA: “THE MOVEMENT THAT RAFAEL CORREA LEADED

WAS FROM AN UNDETEED SOCIAL PARTICIPATION”

\section{Sofia Bernat}

sofiabernat@gmail.com | https://orcid.org/0000-0002-7178-3829

\author{
Sol Logroño \\ sol12lgr@gmail.com | https://orcid.org/0000-0002-5807-5748
}

Emiliano Sánchez Narvarte

emiliano.sanchez@perio.unlp.edu.ar | https://orcid.org/0000-0002-5407-3681

Instituto de Estudios Comunicacionales en Medios, Cultura y Poder Aníbal Ford

Facultad de Periodismo y Comunicación Social

Universidad Nacional de La Plata | Argentina

\begin{tabular}{|c|c|}
\hline Resumen & Abstract \\
\hline El sociólogo y profesor de la Universidad & The sociologist and professor at the \\
\hline Politécnica Salesiana de Ecuador, & Universidad Politécnica Salesiana \\
\hline reflexiona en esta entrevista & of Ecuador, reflects in this interview \\
\hline sobre el proceso que posibilitó la & about the process that made possible \\
\hline conformación y la llegada al gobierno & the conformation and arrival to the \\
\hline de Alianza País, el rol de los medios & government of Alianza País, the role \\
\hline y la participación ciudadana. & of the media and citizen participation. \\
\hline
\end{tabular}

Palabras clave | Ecuador, Alianza País, Correa, participación ciudadana

Keywords | Ecuador, Alianza País, Correa, citizen participation 


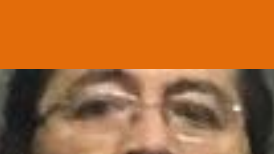

Entrevista a René Unda Lara

\title{
"EL MOVIMIENTO QUE LIDERÓ RAFAEL CORREA
}

\section{FUE DE UNA PARTICIPACIÓN SOCIAL INÉDITA"}

\author{
Por Sofia Bernat, Sol Logroño \\ y Emiliano Sánchez Narvarte
}

René Unda Lara es doctor en ciencias sociales, sociólogo y profesor investigador en la Universidad Politécnica Salesiana de Ecuador. Entre sus principales indagaciones, se encuentran sus trabajos sobre movilización social, activismo y acción colectiva juvenil. En esta entrevista, destaca el proceso que posibilitó la conformación y la llegada al gobierno de Ecuador de Alianza País.

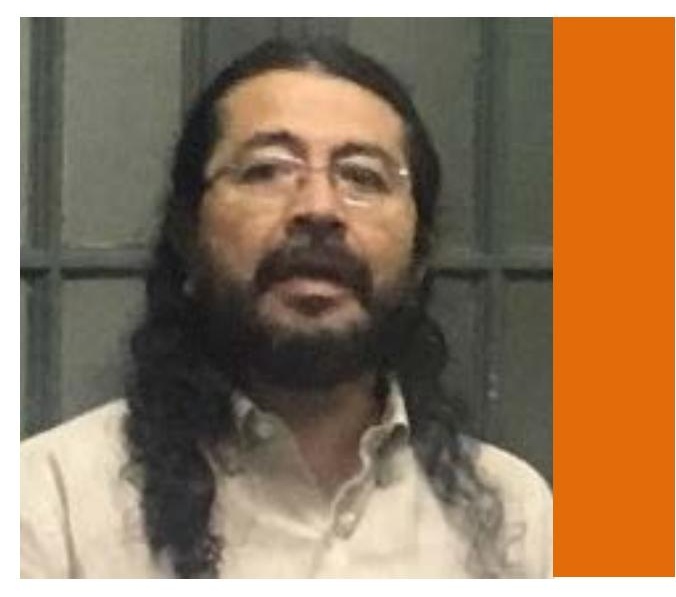




\section{¿Cómo se gestó el proceso que en la historia ecuatoriana pareciera que comenzó con el movimiento Alianza País?}

El proceso no puede explicarse al margen de un vistazo a lo que ha sido, en términos muy generales y gruesos, el retorno a la democracia. Si hacemos un corte típicamente sociológico, Ecuador fue uno de los primeros países en los que se propuso el retorno de las dictaduras tuteladas de los años setenta, no solo por la convergencia de fuerzas politicas internas, sino porque todas las estrategias geopoliticas hemisféricas así lo determinaban.

En 1979 recuperamos la democracia a través de un referéndum. En 1971 tomó el poder Guillermo Rodríguez Lara, hasta 1976, y fue un período de transformación estructural porque Ecuador exportó su primer barril de petróleo de forma soberana. El país pasó de una economía precarizada, asentada en la dinámica de reproducción y de estrategias socioeconómicas casi excluyentemente rurales, a una economía que dependía de los ingresos petroleros. Esa economía primario extractivista fue la que permitió la creación - no solo la incorporación - de amplias capas medias, de sectores medios que accedieron a la educación en una matriz general de modernización socioeconómica latinoamericana.

\section{¿Cómo se produjo el retorno a la democracia?}

La vuelta a la democracia supuso que las fuerzas políticas que estaban acumuladas previo a la dictadura, y que fueron emergiendo durante esos años, encontraran los canales democráticos para presentarse al referéndum con mucha expectativa. En realidad, diría que de una forma bastante ingenua, en la medida en la que no se analiza con la debida cautela el orden del concierto internacional, la geopolítica, y, en cierta medida, caemos en la ilusión de creer que nosotros construimos nuestra democracia de forma autónoma, autodeterminada. 
En ese sentido, fueron siempre las derechas las que llevaron la batuta y las que plantearon, ya desde el mismo referéndum, el carácter de esa democracia. Una democracia fundamentalmente representativa, de corte decididamente liberal y con muy poca perspectiva republicana. Eso, en un contexto en el que la estructura socioeconómica se estaba desatando de sus dos o tres siglos de historia hacendataria, de la estructura latifundista y oligopólica que no había posibilitado las condiciones para una burguesía nacional moderna. Eran grupos oligárquicos depredadores: por un lado, los terratenientes serranos y con ciertas proyecciones industriales que no habían llegado a cristalizar; por otro, los exportadores costeños. Fue un momento fuerte, porque se des-constituyeron las centrales obreras. Empezó un proceso de debilitamiento de la central única de trabajadores de la Confederación Ecuatoriana de Organizaciones Sindicales Libres (CEOLS), la central donde se agrupaban los sindicatos. Pero también fue un momento interesante, porque fue el momento identitario reivindicativo del movimiento indigena. ${ }^{1}$

Con el retorno a la democracia se sucedieron una serie de gobiernos de distinto signo pero con una matriz común: un modelo de acumulación basado en la economía de exportación primaria manejado por elites que no tenían el menor sentido de lo nacional, por jerarcas y caciques locales y por especuladores. Ese ha sido el patrón común de la dinámica de reproducción económica.

\section{En lineas generales, ¿cómo se podrian caracterizar los procesos socioeconómicos ecuatorianos entre las décadas del ochenta y del noventa?}

Un primer ciclo político se desarrolló entre 1979 y 1996. Entonces, un gobierno de derecha y represivo, que tenía una suerte de intención de redistribución de la riqueza, se topó con la coyuntura internacional, con el Consenso de Washington, un plan de ajuste estructural que significó no tanto flexibilización del mercado financiero —en el caso ecuatoriano en esos años- sino, sobre todo, políticas anti-trabajo, anti-laborales, privatizaciones, flexibilización 
laboral, desregulación del mercado financiero: los ejes estructurantes del conjunto de politicas neoliberales que se instauraron en el país hasta 1996.

Ese año, el país movilizado, desencantado de la democracia, vivió un proceso acelerado de des-consolidación democrática. Si bien se trató, también, de una época de auge de movimientos sociales, por una serie de razones no necesariamente correspondientes a la dinámica interna, sino también por la cooperación internacional, un momento muy oenegista. Estaba el Consenso de Washington, con la lucha contra la pobreza y con algunos de esos clichés que son espejismos, como la lucha contra la pobreza pero nada contra el modelo de acumulación. La sociedad se movilizó e irrumpió la figura de Abdalá ("El loco») Bucaram, que venía de ser alcalde de Guayaquil. Bucaram llegó y ganó las elecciones de 1996; se posicionó y empezó una política muy esquizoide, en el sentido de que era un discurso nacional, popular, populista, pero un populismo más de derecha. ${ }^{2}$ Duró seis meses en el gobierno. Lo destituyeron, por demencia, en febrero de 1997.

Entre 1996 y 2006 se desarrolló el segundo ciclo, con crisis, derrocamientos políticos, ${ }^{3}$ en un proceso trazado por la exclusión y por el autoritarismo que se tradujo en racismos. Entonces, en 2002 ganó Lucio Gutiérrez con una propuesta nacionalista. Al día siguiente, atendió a los Estados Unidos y se puso a las órdenes de George Bush. Era el mejor amigo de los Estados Unidos. Y nos traicionó, porque a él se habían plegado el movimiento indígena, las fuerzas de izquierda, y el contendiente, el adversario político a ganar era la derecha social cristiana. El país se habia unido. Creo que ha habido una vocación progresista del país pero que se topa con los límites propios de sus figuras presidenciales y con los intereses y las presiones que se imponen más estructurados históricamente. Gutiérrez fue derrocado en abril de $2005 .{ }^{4}$ 


\section{¿Cómo fue la conformación de Alianza País y el ascenso a la presidencia de Rafael Correa?}

2006 fue un año puente en el que empezaron a reconstituirse las fuerzas sociopolíticas; entre ellas, Alianza País. Para ese entonces, Alianza País venía constituyéndose como movimiento indígena, como una congregación de 222 movimientos políticos a escala nacional que constituyen lo que se llama Movimiento País. Hubo una serie de internas, hasta que el 20 de enero de 2007 se definió la candidatura de Rafael Correa. Su figura fue creciendo en la medida en la que fue caminando la calle. Entre enero, que tenía una intención de voto de $3,2 \%$, y la primera vuelta electora de abril había crecido a $29 \%$, lo que lo ubicaba en segundo lugar; y hacia las elecciones de noviembre de 2006 llegó con cerca de 60\%.

En enero de 2007 surgió la Revolución Ciudadana con tres propuestas: 1. Lo constitucional, sobre lo que plegaron las fuerzas progresistas de izquierda, indígenas y que lo lleva adelante. Un proceso complejo e inéditamente participativo. La elaboración de la Constitución del Buen Vivir. 2. Un nuevo sistema político que es la lucha contra la partidocracia. Llama a nuevas inscripciones, hay nuevas reglas democráticas. 3. El Buen Vivir. Esto tiene implicaciones importantes. Se sostiene el desarrollo y el crecimiento. No se puede borrar ese modelo, ese paradigma rápidamente. Pero más que el desarrollo convencional, basado en el crecimiento económico, se va suspendiendo de a poco para ser desplazado por la concepción del Buen Vivir, basada en creencias de universos andino-ancestrales. Entonces, proceso constituyente, nuevo sistema politico y Buen Vivir son los puntales que organizan la revolución ciudadana. Ese es el tema de la reconfiguración del momento inicial de la última década y sobre eso se montan consultas, procesos electorales y una creciente legitimación del proyecto de la revolución ciudadana y, sobre todo, de la figura del presidente Correa. 


\section{¿Se encontró con muchas resistencias el proceso de reforma de la Carta Magna?}

En realidad, por los resultados electorales, no. La elección de asambleístas la ganó Movimiento País, con el 80\%. Y también con el $80 \%$ ganó la carta constitucional. Esta votación masiva de Alianza País correspondió a un primer momento de este ciclo y no dejó de tener opositores feroces. No han participado en igualdad de condiciones pueblos afros, pueblos indigenas. Persiste una mirada elitista. De todos modos, constitucionalistas que en el antiguo régimen eran constitucionalistas consagrados y voceros de los sectores que iban quedando ya se sentian desplazados del escenario político. En este aspecto hubo mucha resistencia, pero focalizada. Con voces influyentes, pero cada vez más arrinconadas.

\section{¿Qué actores participaron en estos procesos?}

Desde siempre, los trabajadores, pese al debilitamiento sindical, estaban ahí. También el movimiento indígena, un movimiento protagonista de la política nacional en los ochenta y en los noventa. El debilitamiento del movimiento indígena se comenzó a evidenciar no tanto a finales de los noventa como, más bien, en sus alianzas con Lucio Gutiérrez. ${ }^{5}$ Si se analizan los tres componentes del sistema político (sistema de representación, sistema de gobierno y sistema de administración público), se observa que el sistema de representación era cooptado por intereses particulares, intereses de los grupos dominantes - lo cual no es ajeno a la dinámica regional ni a la propia historia del sistema politico ecuatoriano-, pero que plantean un nuevo escenario. Ese nuevo escenario era la absoluta de-sintonización con la movilización y con las demandas populares de aquel momento. El movimiento indígena, los sectores de estudiantes de izquierda, sobre todo, y los movimientos afrodescendientes. Pero era una ciudadanía movilizada, no organizada. La clase media ilustrada de Quito tenía esperanza de un gobierno progresista. Por eso es importante remarcar aquí la presencia del 
ciudadano común no organizado, porque esto tiene implicaciones fuertes en términos teóricos y prácticos. Es decir, en este aspecto, no creo en la cuestión de una sociedad civil como se ha tratado de plantear desde algunos postulados teóricos. No hay sociedad civil. Lo que hay es una sociedad movilizada, al menos en este momento.

\section{¿Cómo se dan esos procesos de participación?}

Es un nivel de participación que desde algunos intentos de teorización hemos llamado "participación movilizada fractal, granular, atomizada", pero que no deja de tener eficacias politicas en términos de derrocamientos presidenciales, en términos de llevar adelante propuestas que finalmente configuran el programa politico de Alianza País en un determinado momento. Con estas características, Correa se constituye en lider y se desatan los diez años con tres ciclos políticos claramente diferenciables: 2006-2008, es el momento pre-constituyente, el eje estructurador; 2008-2012, durante el cual se viven la bonanza y la contundencia de Correa y el marcado desplazamiento de los sectores que históricamente habian mangoneado el país; y 2012-2016, cuando empieza el "cuesta arriba" de la Alianza País.

No solo a nivel regional sino a nivel global comenzó la baja del precio del petróleo y, sobre todo, las dinámicas de orden interno en cuanto a factores de cultura politica y de una sociedad que había ganado derechos y que, en un momento determinado, vio que esos derechos tenían un límite. Clases medias que se habían acostumbrado a salarios muy buenos, a una economía basada en el consumo, pero con muy poca base productiva. Empezaron los recortes, las restricciones, los obstáculos para los viajes de turismo. Una clase media bastante arribista por su propia tradición y que, en cuanto a frustración de expectativas, vio que eso se empezaba a configurar de esa manera. Porque ya no se podía consumir como antes, porque se percibía una orientación al autoritarismo, porque "el Estado mete las narices en todo"... Pero, sobre todo, no hay que desconocer las 
fuerzas estructurales que de alguna forma han estado presentes siempre - con particular fuerza en la sociedad desde los años cincuenta-, y que son las empresas privadas de comunicación.

Es en este contexto, y en el proceso de reforma institucional del Estado, que apareció la propuesta de ley de comunicación. Porque esa dinámica es una dinámica particular por su fuerza relativa, en comparación a otras fuerzas. Las mismas fuerzas de representación política de la derecha no serían nada en términos de resultados electorales sin el soporte de las empresas privadas de comunicación. Empresas que tienen, además, lo que se llama "intereses vinculados". Este es el primer punto de la ley de comunicación.

El 8 de febrero de 2011 Rafael Correa lanzó la propuesta de decreto y la consulta popular en la que preguntaba si la población estaba de acuerdo con que los dueños de medios fueran, simultáneamente, dueños de bancos y de otros negocios. Correa hablaba en vivo los sábados y daba clases para explicar al pueblo popular el significado de los "créditos vinculados": una relativa ausencia de mediaciones por las exigencias y por el vértigo del proceso político, frente a unas fuerzas que lo acusaban constantemente de autoritario y en un marco en que era atacado en forma permanente.

Por ejemplo, en el momento pre-constituyente, cuando se cocina la constitución del Buen Vivir, sucedieron cosas desde distintos sectores y actores. La Iglesia, la cúpula eclesial, que tiene una fuerte incidencia en la población ecuatoriana, especialmente en Quito, en Guayaquil, en Cuenca, difundía campañas de modo abierto, público $\mathrm{y}$ persistente en torno a que el objetivo de la constituyente era confiscar la propiedad privada: "Si tú tienes dos casas, te van a quitar una, al menos. Si tienes dos o tres autos, te van a dejar solo con uno. $\mathrm{Y}$ tus hijos van a ser adoctrinados y formados en la matriz castro-chavista. Luego, la lógica de este comunismo que nos quiere implantar Rafael Correa". Se resaltaban exabruptos de Correa gritando. Y eso se empezó a instalar progresivamente. Pero Correa llegaba los sábados y discutía esa imagen. 
En la Argentina hubo una incorporación de sectores intelectuales al debate público en apoyo al proyecto político. En el caso ecuatoriano, ¿se incorpora también una capa intelectual? ¿Existe una discusión sobre el rol de los medios?

Hay una organicidad, desde luego. No diría una organicidad estrictamente intelectual, a secas. Es, también, una organicidad de activistas, de luchadores populares, de políticos reciclados, incluso lo es Alianza País en la pluralidad de su constitución, personas que eran de derecha y que en algún momento se reconvirtieron, o que son de derecha pero que tienen una mirada interesante en materia de turismo, de dinámicas internacionales.

Correa no es un líder análogo a la figura de Chávez. Mantiene ciertas distancias, pero pliega finalmente al eje regional, considerándose, primero, como un ciudadano más, pero que tiene que llevar adelante el proceso; segundo, es un cristiano de base, pliega mucho a la teología de la liberación e invitó a sus referentes desde el principio. Pero, a su vez, no habló en ningún momento durante el proceso constituyente del socialismo del siglo XXI sino que se autodefinía como un gobierno nacional y popular.

Ciertos académicos participamos durante la primera fase como ciudadanos en las asambleas barriales, en aquel momento de efervescencia. Sectores de la intelectualidad, sectores académicos funcionaron no tanto en la lógica de sectores orgánicos sino como contribuciones y de ahí fue decantándose el equipo de acompañamiento duro de Correa que, fundamentalmente, fue un núcleo duro de economistas de la Universidad Católica, que en los años noventa fueron parte de Liberación Nacional, un partido progre, izquierdista, pero no una izquierda roja sino más bien de ciertas burguesías progresistas. Ese es el núcleo duro. 
Todo esto favoreció la construcción de esta nueva institucionalidad. Fue una aplanadora y una hecatombe para las derechas. Las derechas se rearmaron, sobre todo, vinculadas a través de lo que Correa llama la "prensa mediocre y corrupta". Las sabatinas no fallaron ni un solo sábado, de 10 de la mañana a 2 de la tarde; en ellas hubo pedagogía política, explicación al pueblo. No podían con él, porque además de que era un profesor para el pueblo tenía su particular pedagogía y comunicación política. Su palabra tenía encantada a la gente. Usaba un lenguaje cotidiano, porteño, extrovertido, toquetón, buen pana, hablaba con malas palabras. En las sabatinas, Correa tenía un arma para contrarrestar todo lo que de lunes a viernes se planteaba como agenda: destruirlo por cualquier medio, diciendo que la Revolución Ciudadana no estaba funcionando, que cómo se podía gastar tanta plata.

De todos modos, empezó a producirse un desencantamiento progresivo de sectores medios, rojos, radicales que, imbuidos de su lógica abstracta de la realidad, sostienen que el país no puede reducirse a carreteras, que no alcanza si no se transforma de manera revolucionaria. Sin embargo, al trabajar con comunidades campesinas, se ve que por primera vez en la historia del país sus productos podian salir, fuera o no fuera invierno, que se podía ir al páramo en camioneta y cargar las cebollas, los productos agrícolas y sacarlos al mercado, que se podía tener un plan de crédito que no habia funcionado para los sectores agropecuarios campesinos indigenas durante dieciocho años, como el programa Fomento del Desarrollo Rural y Producción Agropecuaria (FODERUMA).

El momento constituyente fue de una participación ampliada inédita, una explosión brutal de participación. Existian canales. Si no eras de ninguna organización, funcionaban a modo de secretarias itinerantes de las comisiones y se articulaban con otras individualidades. Esta eclosión participativa es la que explica que la aprobación de la Constitución ganara con el 80\%. Luego, la Constitución se estableció y comenzó un período de intervención del Estado, legitimado, cada vez más creciente. Antes lo público era una cuestión negativa: los hospitales, el sistema de salud, la educación. Lo público era 
considerado como "cosa de pobres". Pero comenzó una fuerte revalorización de lo público, eran las mejores escuelas, los mejores hospitales, las mejores carreteras, sin peaje. Todo ese proceso que se vivió tan aceleradamente hacia 2012 también fue combatido, sobre todo, con una agenda sistemática de medios de comunicación privados.

\section{Notas}

1 El movimiento indígena estaba articulado al Estado desde 1938 con la Ley de Comunas. Esto cristalizará en términos politicos hacia mediados de los noventa con Pachakuti, el brazo político del movimiento indígena.

2 Bucaram incorporó a Domingo Cavallo como asesor económico, específicamente, para la conversión monetaria. En 1999 se dolarizó la economía ecuatoriana.

3 Entre el 21 y el 22 de enero de 2000, y que produjo la destitución del presidente Jamil Mahuad.

4 Existía un descontento en la población por su impronta autoritaria y por su relación con el Fondo Monetario Internacional. Se produjeron movilizaciones -encabezadas por clases medias quiteñas pero con acompañamiento indígena-, que tenían un componente racista.

5 Lucio Edwin Gutiérrez Borbúa, nacido en la ciudad de Quito el 23 de marzo de 1957, fue presidente de Ecuador del 15 de enero de 2003 al 20 de abril de 2005. 